\title{
Tackling delays in patients requiring chest X-rays on the acute medical take
}

\author{
Authors: Adam Bharmal ${ }^{A}$ and Tehmeena Khan ${ }^{B}$
}

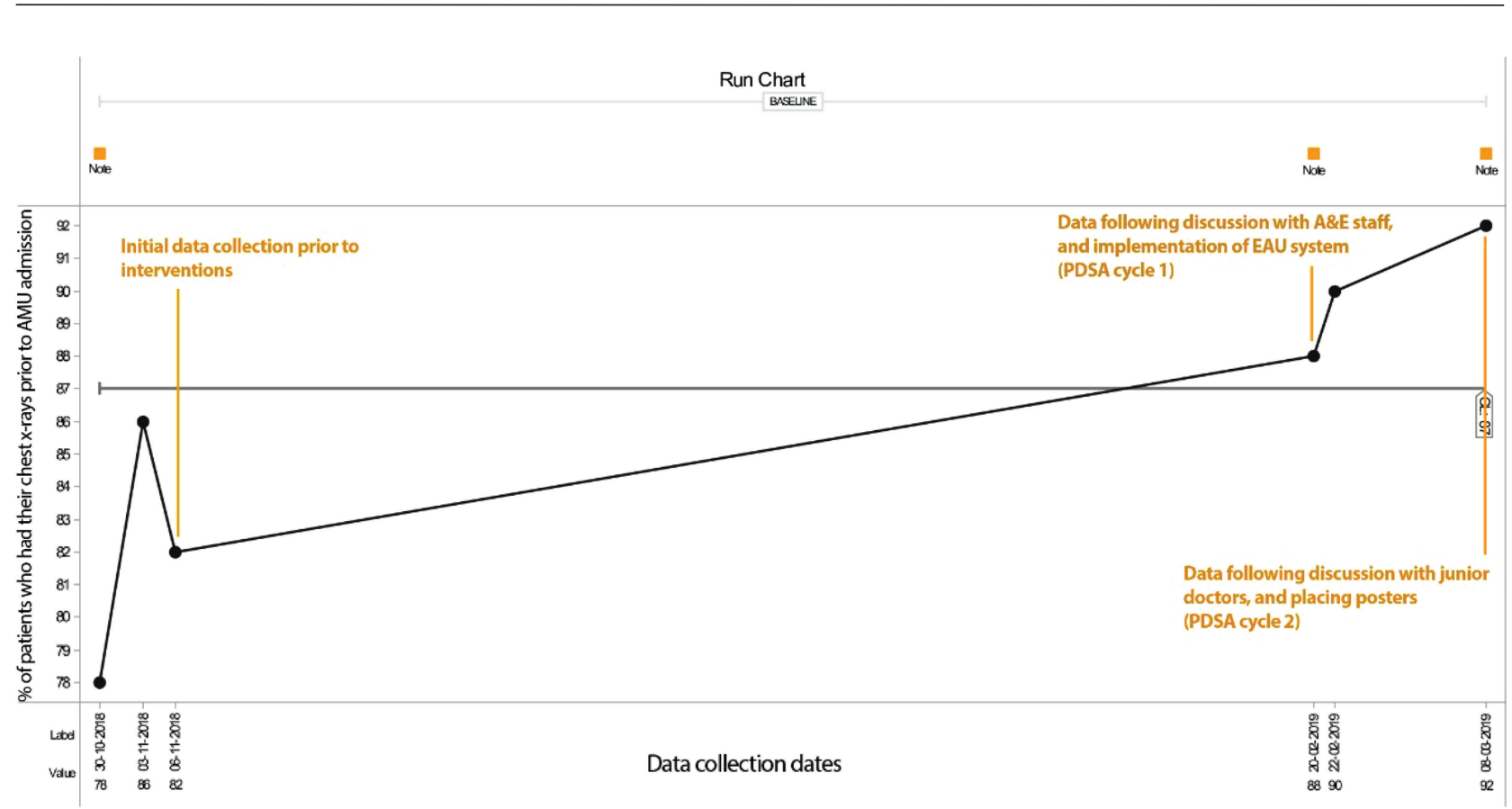

Fig 1. Run chart demonstrating the improving proportions of patients receiving clinically indicated chest X-rays prior to their acute medical unit admission, with labels detailing timing of interventions and the corresponding plan, do, study, act cycle to which they belong.

\section{Introduction and aim}

Chest X-rays form a vital part of the initial assessment and management of patients seen by medical practitioners. ${ }^{1}$ During the acute medical take at University College Hospital (UCH), patients are referred to the medical team by the emergency department (ED) team or via their general practitioner. Due to logistical arrangements, patients may be transferred to the acute medical unit (AMU) without the chest X-ray that they require as beds become available. As a result, patients would then be transferred to the X-ray department at a later time or date to have this crucial investigation when it could have been performed in ED at the time of admission. This can lead to

Authors: ${ }^{A}$ Royal Free London NHS Foundation Trust, London, UK; ' ${ }^{B}$ nniversity College London Hospitals NHS Foundation Trust, London, UK delays in diagnosis and management, as well as unnecessary disruption for the patient. This quality improvement project was designed to tackle these delays in obtaining chest X-rays between October 2018 and March 2019.

\section{Method}

Electronic health records of 122 patients were analysed preintervention to determine the proportion that did not receive a chest X-ray prior to admission to the AMU. The delay in patients who had the X-ray after AMU admission was recorded. Two plan, do, study, act (PDSA) cycles with interventions focussed around improving communication between ED staff, acute medicine doctors and radiographers were performed. A further 135 patient records were analysed post-intervention to determine if there was any increase in the proportion of patients receiving an $\mathrm{X}$-ray prior to admission and if that corresponded to a reduced delay. 


\section{Results}

By the second cycle, the percentage of patients receiving their clinically indicated chest X-ray prior to admission to the AMU rose from $82 \%$ to $92 \%$, with delay time reducing from 2 hours and 22 minutes per patient to 42 minutes per patient (Fig 1).

\section{Conclusion}

The results given above translate to reduced disruption for patients ${ }^{2}$ and reduced delays in diagnosis and management for patients admitted under medicine at $\mathrm{UCH}$.

\section{Conflicts of interest}

None declared.

\section{References}

1 British Thoracic Society. Guidelines for the management of community acquired pneumonia in adults update 2009. BTS, 2009. www.britthoracic.org.uk/document-library/clinicalinformation/ pneumonia/adultpneumonia/a-quick-reference-guide-btsguidelines-for-themanagement-ofcommunity-acquiredpneumonia-in-adults/ [Accessed 02 December 2018].

2 Naithani S, Whelan K, Thomas J. Hospital inpatients' experiences of access to food: a qualitative interview and observational study. Health Expect 2008;11:294-303. 